\title{
Neural Synchrony-Monitoring Wireless Brain Implant for Intractable Epilepsy Neuromodulation
}

\author{
Karim Abdelhalim, Hamed Mazhab Jafari, Larysa Kokarovtseva $\dagger$, Jose Luis Perez Velazquez $\dagger$, Roman Genov \\ Department of Electrical and Computer Engineering, University of Toronto, Canada; Email: roman@eecg.utoronto.ca \\ $\nmid$ Brain and Behavior Programme and Division of Neurology, Hospital for Sick Children, University of Toronto, Canada
}

\begin{abstract}
A validation of a closed-loop system-on-chip (SoC) for epilepsy treatment is presented. A $12 \mathrm{~mm}^{2} 0.13 \mu \mathrm{m}$ CMOS SoC provides the functionality of neural recording, neural stimulation and early seizure detection based on the bivariate phase synchrony estimation algorithm. The SoC can operate in a closed loop, has 64 neural recording and 64 neural stimulation channels, and dissipates $1.4 \mathrm{~mW}$ and $1.5 \mathrm{~mW}$ across 64 channels for neural recording and stimulation, respectively. Two in vivo experiments with freely moving rats based on a non-convulsive and convulsive seizure model are presented. The first in vivo experiment validates the results by comparing the output of the SoC with a SIMULINK model of the implant. The second in vivo experiment validates the $\mathrm{SoC}$ in early seizure detection and as a closed-loop intractable epilepsy treatment with 80 percent efficacy. Long Evans rats are used for all experiments. All data are cross-validated by a conventional benchtop amplifier.
\end{abstract}

\section{INTRODUCTION}

Approximately 50 million people worldwide are epileptic. Epilepsy is the third most common neurological disorder following stroke and Alzheimer's disease, but it imposes higher costs on society than stroke does. Approximately 30 percent of epileptic patients suffer intractable epilepsy in that the symptoms do not respond to medication. Half of these individuals will ultimately be deemed candidates for surgical resection of the source of the seizures (i.e., the seizure focus), with its own risks. Preoperative epilepsy monitoring with implanted electrode grids is done to localize the focus, and to ensure that the resection can be accomplished without producing neurological deficits. Resection of the focus in well assessed patients portends an 80 percent chance of them being rendered seizure-free. The remainder of all the patients can only be treated with vagus nerve stimulation [1] and deep brain stimulation [2], both open-loop and of limited benefit. None of these therapies consider the nonlinear dynamics of dysfunctional brain activity exhibiting themselves in changes in neural synchrony, or utilize an automated therapeutic feedback approach such as the one employed in an experimental commercial implant currently under development [3].

Epileptic seizure onsets are often characterized by specific precursors including increased fluctuations in phase synchrony and subsequent increased brain synchronization [4]. There are many studies that have investigated the higher synchrony fluctuations associated with epileptiform activities which show that phase synchrony fluctuation is among the best seizure precursors [5]. Our approach is to implement a novel implantable technology that wirelessly monitors online the ab-

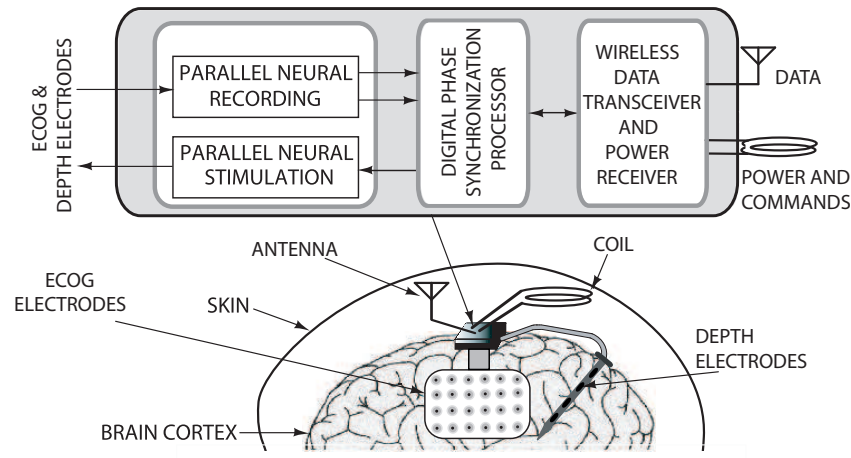

Fig. 1. Wireless closed-loop neural recording and stimulation SoC for intractable epilepsy treatment.

normal neuroelectrical phase synchrony of epileptic seizures in the brain and dynamically aborts them by means of electrical neurostimulation.

The form-factor novelty of the presented approach is derived from the fact that the recording and processing of neurological signals needed to detect epileptic seizures accurately typically requires bulky electrophysiological equipment. Furthermore, significant computing resources are also required to enable online real-time early seizure detection which can then trigger external electrical stimuli. Design of such instruments has been a significant research direction around the world [6], [7], but to date, a closed-loop electrical neurostimulator utilizing a high-efficacy early seizure detector and implemented as a monolithic system-on-chip (SoC) has not been engineered.

A generic block diagram of the envisioned wireless neural stimulator with a closed-loop feedback is depicted in Figure 1. The bidirectional system is connected to the brain through a set of electrodes and is implanted under the skull. Energy is delivered wirelessy through inductive coils and recorded data are sent by an RF wireless transmitter. The key building blocks for the implantable SoC are neural recording, signal processing for early seizure detection, neural stimulation for seizure abortion and a wireless interface.

In this paper we present in vivo results validating the utility of such a closed-loop SoC (presented in detail in [8]) in treating intractable epilepsy. The $\mathrm{SoC}$ operates as a neural vector analyzer to compute the magnitude and phase of neural inputs and to estimate the phase synchrony between a pair of neural inputs. The magnitude, phase and the phase synchrony 
features are used to trigger the neural stimulator to abort or cancel a seizure. The rest of the paper is organized as follows. Section II describes the closed-loop system and the prototype SoC. Section IV presents in-vivo results on rats to validate the closed-loop implantable SoC.

\section{System-ON-ChIP VLSI ARCHITECTURE}

The early seizure detection algorithm is based on computing the magnitude, phase and phase synchrony of neural signals in specific frequency bands in the neural signal spectrum. When the computed phase synchrony increases above a programmable threshold in a moving average time window, a seizure is detected. The seizure detection algorithm is described in detail in [9].

The functional diagram of the implantable SoC is depicted in Figure 2 and its micrograph implemented in a standard 1P8M IBM $0.13 \mu \mathrm{m}$ CMOS technology is shown in Figure 3. It consists of 64 neural recording and 64 neural stimulator channels. Two pairs of neural inputs get amplified and then bandpass filtered using a high-Q switched capacitor bandpass filter. The center of the bandpass filter is set to a specific band in the neural signal spectrum.

For offline human experiments the BPF was set to $16 \mathrm{~Hz}$ or $32 \mathrm{~Hz}$. For in vivo rat experiments, the bandpass filter was set to either $4 \mathrm{~Hz}$ or $8 \mathrm{~Hz}$. Next, the two inputs are passed to two pairs of mixed-signal FIR filters, an all-pass and Hilbert FIR filter, to extract the (in-phase) real (I) and (quadrature phase) imaginary $(\mathrm{Q})$ components. The FIR multiplication is performed in the analog domain within the energy efficient SAR ADC. The add-and-delay blocks of the FIR filters are implemented in the digital domain. This FIR implementation minimizes the area and power dissipation as described in [10]. Next, the real and imaginary components are fed to the on-chip 10-bit CORDIC-based processor that computes the phase synchronization value (PLV) as well as the instantaneous phase $(\phi)$ and magnitude (MAG). A threshold detector can be programmed to trigger the neural stimulator in the feedback to form a closed-loop system. To complete the system all digital data can be transmitter wirelessly using an on-chip 3.110.4GHz UWB transmitter. The closed-loop seizure detection $\mathrm{SoC}$ is described in detail in [8].

The analog front-end has a noise floor of $3.1 \mu \mathrm{V}_{r m s}$ between $1 \mathrm{~Hz}$ and $100 \mathrm{~Hz}$, a CMRR of $75 \mathrm{~dB}$, a gain of $60 \mathrm{~dB}$ and a THD of 0.3 percent for a $1 \mathrm{mV}$ input. In the recording feed-forward path which including the amplifiers, switchedcapacitor (SC) filters, finite impulse response (FIR) filters and the processor, the SoC dissipates $1.4 \mathrm{~mW}$ for 64 -channels from a $1.2 \mathrm{~V}$ supply. Such low power is achieved by performing signal processing in the mixed-signal VLSI domain [10]. In the stimulation mode, the $\mathrm{SoC}$ dissipates $1.5 \mathrm{~mW}$ from a $3.3 \mathrm{~V}$ supply. The magnitude extraction is accurate to below $8 \mu \mathrm{V}_{r m s}$ and the phase extraction operates properly with amplitudes as low as $10 \mu \mathrm{V}_{r m s}$. The neural stimulator can provide biphasic currents with 8-bit resolution between $10 \mu \mathrm{A}$ and $1.2 \mathrm{~mA}$, with 4-bit duty cycle control, frequencies up to $5 \mathrm{kHz}$ and a voltage compliance of $2 \mathrm{~V}$.

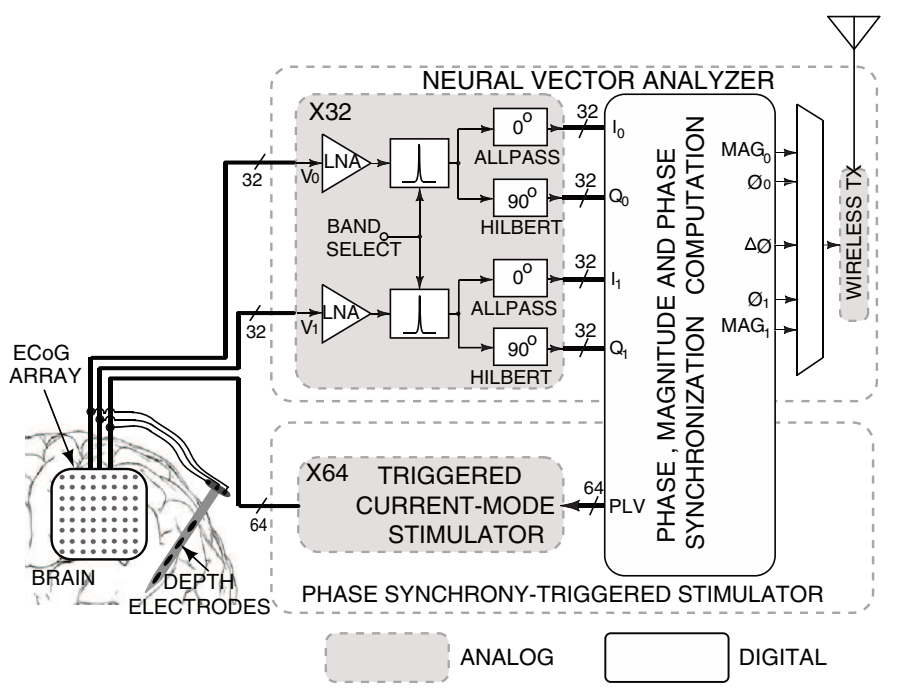

Fig. 2. Functional diagram of the mixed-signal VLSI SoC.

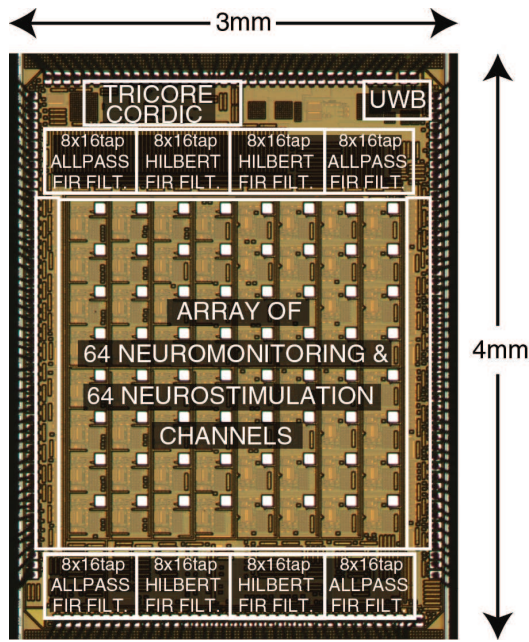

Fig. 3. Micrograph of the $4 \mathrm{~mm} \times 3 \mathrm{~mm} 0.13 \mu \mathrm{m}$ CMOS SoC.

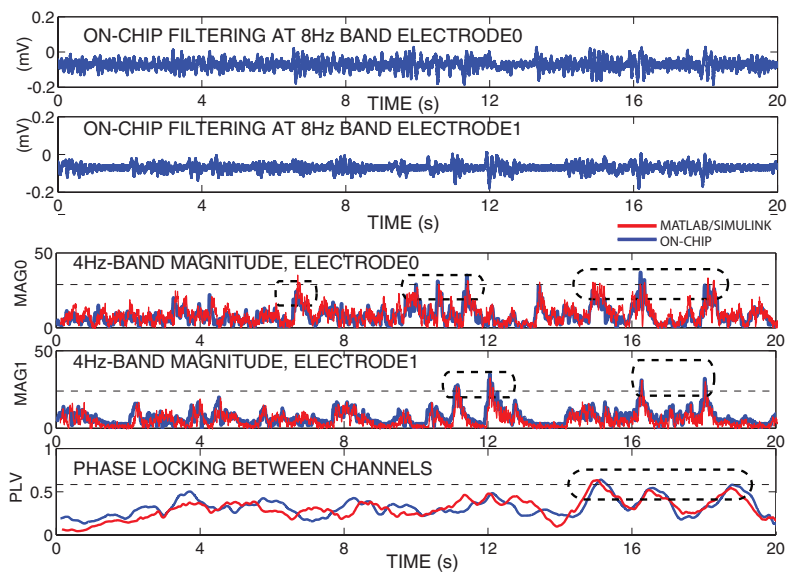

Fig. 4. Example of magnitude and phase locking value extraction on a freely moving rat injected by GBL. Comparison of an offline SIMULINK model with online real-time SoC. 


\section{Offline Human early Seizure Detection}

The efficacy of the phase synchronization processor VLSI architecture in early seizure detection was verified on the intracranial EEG database from the international seizure prediction project from University of Freiburg [11]. A 70 percent detection rate is obtained when the false positives are set to the rate of 0.6 false positives per hour and the detection rate approaches 100 percent when the false positives rate is set to 1.2-2 false positives per hour [9]. This is comparable to other algorithms for early seizure detection, with the key advantage that our approach utilizes a low-power $\mathrm{SoC}$ that computes on line and is implanted, as opposed to existing offline software programs running on bulky computers.

\section{ONLINE In vivo Rodent SEIZure Control}

\section{A. Validation of the Prototype In vivo}

A GBL seizure model was used to validate the neural vector analyzer and the phase synchrony extractor of the $\mathrm{SoC}$ in vivo. The pharmacological agent GBL was injected into the hippocampus of a Long Evans rat (50 to 90 days old) with a dose of $100-120 \mathrm{mg} / \mathrm{kg}$ to induce non-convulsive seizurelike events. After injection of GBL, the magnitude and phase synchrony in the $8 \mathrm{~Hz}$ frequency band both increase as the freely moving rat experiences absence-like seizure activity. The amplified and bandpass filtered (at $8 \mathrm{~Hz}$ ) outputs of two channels are shown in Figure 4 (top two plots). The amplified and bandpass filtered outputs of two channels were processed online in real-time by the $\mathrm{SoC}$ as shown in Figure 4 (bottom three plots). Similarly, the amplified and bandpass filtered outputs were fed offline through a SIMULINK model of the processor (described in [9]) as depicted in Figure 4 (bottom) (highlighted in red). A few points of interest are marked on both plots for comparison. The two peaks on the magnitudes match closely between the SIMULINK model and the SoC. The phase synchrony has two peaks higher than a threshold. Both these peaks match closely between the SIMULINK model and the SoC.

\section{B. Online In Vivo Early Seizure Detection}

A chronic model of a different type of seizure syndrome, that simulates limbic epilepsy in humans, was used. The reason is that most of malignant human seizures are of this type, while absences are considered sort of benign. This model is normally termed in the literature the self-sustained status epilepticus model in rats. It can be caused by injection of kainic acid. Due to the spontaneous nature of the seizures with this model, it can be used to validate the early seizure detector and neural stimulator.

Four bipolar electrodes (Plastics One) were implanted chronically into specific brain areas of Long Evans rats (5090 days old) using a stereotaxic apparatus. The coordinates of the implanted electrodes are summarized in Table I. Three electrodes connect to the closed-loop SoC. These electrodes connect to two recording channels and provide a reference voltage. The other electrode connects to a differential CyberAmp 380 signal conditioner (Axon Instruments),

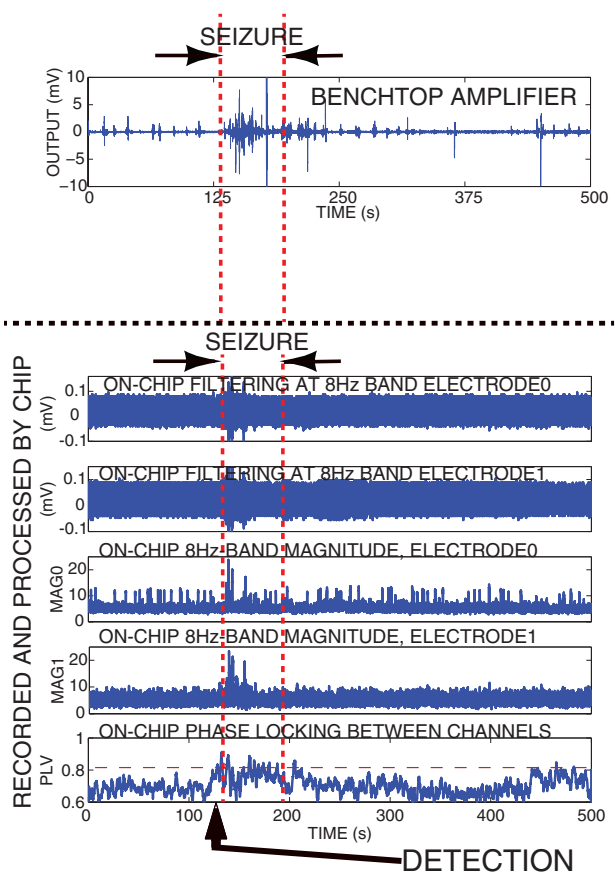

Fig. 5. Example of an early seizure detection example on a freely moving rat in vivo. Seizure triggered by a kainic acid injection at time $\mathrm{t}=-5 \mathrm{~min}$.

TABLE I

Electrode CoORdinates

\begin{tabular}{cccc}
\hline Electrode No. & Bregma (B) & Mediolateral (ML) & Dorsoventral (D) \\
\hline 1 & +2 & -2 & +2 \\
2 & +2 & +2 & +2 \\
3 & -3.8 & -3 & +1.8 \\
4 & -3.8 & +2 & +1.8 \\
\hline
\end{tabular}

and an analog-to-digital converter (MP100) to cross-validate the results. Seizures were identified by examining the rat's electrical activity recorded by the the benthtop amplifier and by observing the rat's seizure-like symptoms, both by trained epileptologists.

Rats were injected with a dose of kainic acid. The administered dose was a $15-20 \mathrm{mg} / \mathrm{kg}$ intraperitoneal injection. The SoC provided voltage amplification and bandpass filtering within selected frequency bands. The information in these frequency bands was then processed by the SoC in real-time on the same chip. The magnitude of two neural inputs and the phase synchrony between two neural inputs were computed by the SoC.

In Figure 5 (top), the raw recording by the benchtop amplifier after offline highpass filtering is shown. The rat had a seizure lasting 60 seconds starting at time $t=130$ seconds. Kainic acid injection was administered at time $\mathrm{t}=-5 \mathrm{~min}$. The SoC processed the $8 \mathrm{~Hz}$ frequency band with the analog output of two neural inputs shown in Figure 5. The real-time magnitude and synchrony computed on-chip in the $8 \mathrm{~Hz}$ frequency band are also shown in Figure 5. The processed magnitude of both inputs increases during the seizure. The synchrony between the two inputs increases above a threshold 10 seconds before the electrical seizure onset, resulting in an early seizure 


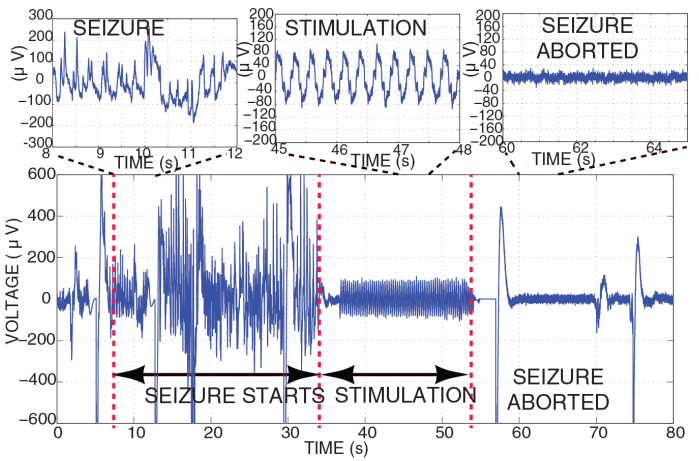

Fig. 6. Successful abortion of a seizure in a rat using the on-chip neural stimulator.

detection. For this rat, on four different seizures the real-time processing of phase synchrony between two inputs yielded early seizure detection of between 4 and 10 seconds before the seizure onset.

\section{Online In vivo Seizure Abortion}

For this case, each channel was configured for both neural recording and stimulation. Once a seizure was identified electrically and visually by seizure-like symptoms, the neural stimulator was invoked by providing a low-frequency $5 \mathrm{~Hz}$, $100 \mu \mathrm{A}$ biphasic current between the same two electrodes. Once the neural stimulation is invoked, the recording and processing capabilities of the SoC are disabled (the SoCs amplifiers are otherwise saturated) and the benchtop amplifier is used to analyze the results.

The first example is depicted in Figure 6 and shows the neural stimulator aborting a seizure instantaneously after the neural stimulator is invoked. The other example, shown in Figure 7, demonstrates closed-loop operation with the $\mathrm{SoC}$ processing the $8 \mathrm{~Hz}$ frequency band. In this case, both the magnitude and synchrony in the $8 \mathrm{~Hz}$ frequency band gradually increase before the seizure for at least 40 seconds. After 6 seconds into the seizure, the neural stimulator was invoked and the seizure symptoms and electrical activity were aborted after 2 seconds. For this rat, the real-time processing of phase synchrony between the two inputs could be used to provide early seizure detection of between 30 and 50 seconds before the seizure onset, but was delayed on purpose to enable seizure visualization in this example. A total of $80 \%$ of seizures were aborted through neural stimulation in four rats tested. A largepopulation rodent study is under way.

\section{CONCLUSIONS}

Validation of epilepsy treatment utilizing a single implantable electronic microchip was presented. Experimental early seizure detection and closed-loop seizure abortion were both demonstrated in vivo on rats. The SoC can compute the magnitude, phase and phase synchrony of 64 neural signal inputs in real time. The $\mathrm{SoC}$ can also operate as a biphasic neural stimulator on up to 64 channels. In vivo experiments on rodents demonstrate a closed-loop treatment for intractable epilepsy with 80 percent efficacy.

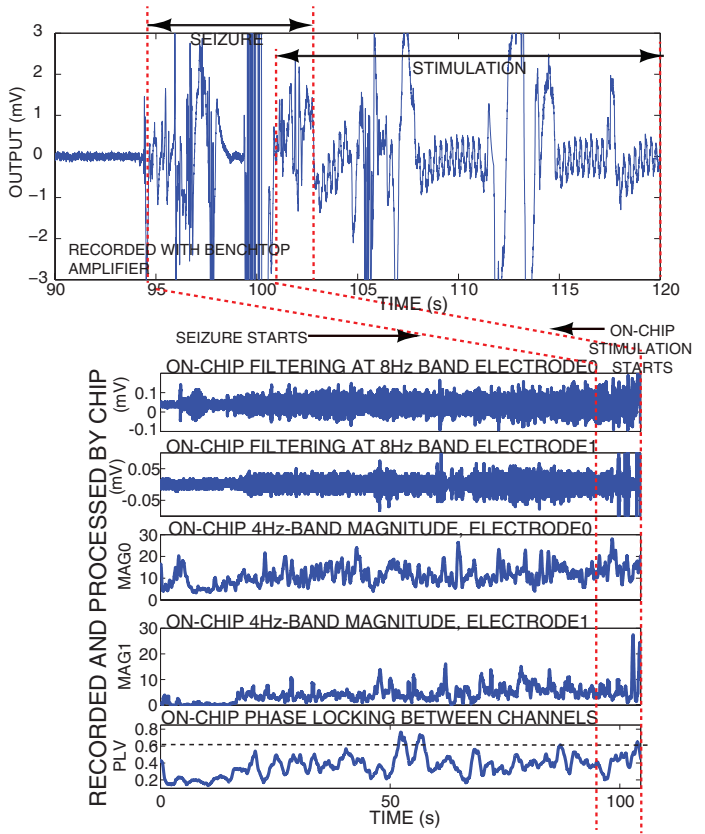

Fig. 7. Example of closed-loop neural stimulation aborting a seizure for a freely moving rat in vivo. Seizure triggered by a kainic acid injection at time $\mathrm{t}=-5 \mathrm{~min}$.

\section{REFERENCES}

[1] Dieter Schmidt, "Vagus-nerve stimulation for the treatment of epilepsy," Lancet Neurol., vol. 1, pp. 477-482, Dec. 2002.

[2] R. Fisher et. al, "Electrical stimulation of the anterior nucleus of thalamus for treatment of refractory epilepsy," Epilepsia, vol. 51, pp. 899-908, May 2010.

[3] F Sun, M Morrell, and R Wharen Jr., "Responsive cortical stimulation for the treatment of epilepsy," Neurotherapeutics, vol. 5, no. 1, pp. 68-74, January 2008.

[4] J. L. Perez Velazquez, L. G. Dominguez, V. Nenadovic, and R. A. Wennberg, "Experimental observation of increased fluctuations in an order parameter before epochs of extended brain synchronization," Journal of Biological Physics, vol. 37, no. 1, pp. 141-152, January 2011.

[5] F. Mormann, R. G. Andrzejak, C. E. Elger, and K. Lehnertz, "Seizure prediction: the long and winding road," Journal of Neurology, Brain, vol. 130, no. 2, pp. 314-333, September 2007.

[6] S. Stanslaski, P. Afshar, P. Cong, J. Giftakis, P. Stypulkowski, D. Carlson, D. Linde, D. Ullestad, A. Avestruz, and Timothy Denison, "Design and validation of a fully implantable, chronic, closed-loop neuromodulation devic with concurrent sensing and stimulation," IEEE Transactions on Neural Systems and Rehabilitation Engineering, vol. 20, no. 4, pp. 410-420, July 2012.

[7] M. Azin, D. J. Guggenmos, S. Barbay, R. J. Nudo, and P. Mohseni, "A battery-powered activity-dependent intracortical microstimulation IC for brain-machine-brain interface," IEEE Journal of Solid-State Circuits, vol. 46, no. 4, pp. 731-745, April 2011.

[8] K. Abdelhalim, H. Mazhab Jafari, L. Kokarovtseva, J. L. Perez Velazquez, and R. Genov, "64-channel UWB wireless neural vector analyzer and phase synchrony-triggered stimulator SoC," IEEE European Solid-State Circuits Conference, September 2012.

[9] K. Abdelhalim, V. Smolyakov, and R. Genov, "Phase-synchronization early epileptic seizure detector VLSI architecture," IEEE Transactions on Biomedical Circuits and Systems, vol. 5, no. 5, pp. 430-438, October 2011.

[10] K. Abdelhalim and R. Genov, "915-MHz wireless 64-channel neural recording SoC with programmable mixed-signal FIR filters," IEEE European Solid-State Circuits Conference, pp. 223-226, September 2011.

[11] International Seizure Prediction Project, "EEG Database," 2010. 